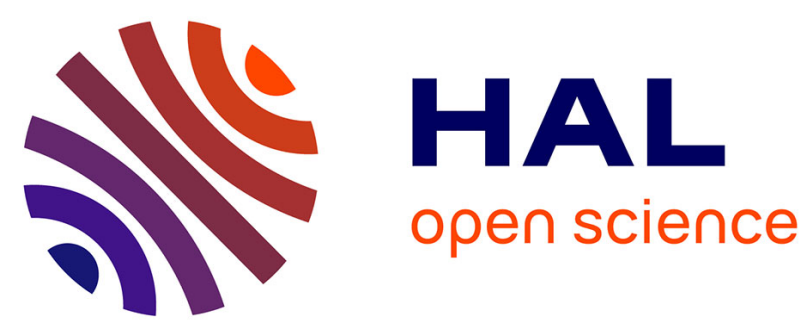

\title{
Efficient third-harmonic generation in a thin nanocrystalline film of $\mathrm{ZnO}$
}

G. I. Petrov, V. Shcheslavskiy, V. V. Yakovlev, Igor Ozerov, E. Chelnokov, W. Marine

\section{- To cite this version:}

G. I. Petrov, V. Shcheslavskiy, V. V. Yakovlev, Igor Ozerov, E. Chelnokov, et al.. Efficient thirdharmonic generation in a thin nanocrystalline film of ZnO. Applied Physics Letters, 2003, 83, pp.3993 - 3995. 10.1063/1.1623948. hal-01618596

\section{HAL Id: hal-01618596 https://hal-amu.archives-ouvertes.fr/hal-01618596}

Submitted on 18 Oct 2017

HAL is a multi-disciplinary open access archive for the deposit and dissemination of scientific research documents, whether they are published or not. The documents may come from teaching and research institutions in France or abroad, or from public or private research centers.
L'archive ouverte pluridisciplinaire HAL, est destinée au dépôt et à la diffusion de documents scientifiques de niveau recherche, publiés ou non, émanant des établissements d'enseignement et de recherche français ou étrangers, des laboratoires publics ou privés. 


\title{
Efficient third-harmonic generation in a thin nanocrystalline film of ZnO
}

\author{
G. I. Petrov, V. Shcheslavskiy, and V. V. Yakovlev \\ Department of Physics, University of Wisconsin-Milwaukee, P.O. Box 413, Milwaukee, Wisconsin 53201 \\ I. Ozerov, E. Chelnokov, and W. Marine \\ Université de la Méditerranée, UMR 6631 CNRS, 13288 Marseille, France
}

(Received 16 May 2003; accepted 8 September 2003)

\begin{abstract}
Nonlinear optical conversion is studied in thin films of wide-bandgap materials. Very high conversion efficiency to the third-harmonic radiation is achieved for an unamplified femtosecond $\mathrm{Cr}^{4+}$ :forsterite laser in a submicron-thick film of a nanocrystalline $\mathrm{ZnO}$ pulsed-laser-deposited on a fused silica substrate. (C) 2003 American Institute of Physics. [DOI: 10.1063/1.1623948]
\end{abstract}

Wide-bandgap semiconductors have recently attracted interest for their applications in solid-state electronics and optics. ${ }^{1,2}$ In particular, zinc oxide $(\mathrm{ZnO})$ is a II-VI semiconductor with a wide bandgap of $3.37 \mathrm{eV}$, and a direct structure at room temperature. ${ }^{3}$ It is especially attractive because of the recent demonstrations of its applicability as a perspective material for near-UV emitters ${ }^{4-6}$ and ultrafast UV light modulators. ${ }^{7,8}$ On the other hand, there is a significant demand for thin-film nonlinear optical materials, which can be easily integrated into an opto-electronic device. ${ }^{1,2,9,10}$ It has been recently pointed out that $\mathrm{ZnO}$ films have a strong nonlinear second-order optical susceptibility $\chi^{(2)}$, and the efficient second harmonic (SH) has been generated in thin films of $\mathrm{ZnO} .{ }^{11,12}$ However, most of the nonlinear optical devices use higher order susceptibility $\chi^{(3)} \cdot{ }^{13}$ II-VI semiconductors fall in the group of materials with a very high nonlinear refractive index $n_{2}$, which has been measured for $\mathrm{ZnO}$ to be $2.3 \times 10^{-12}$ esu (for $\lambda=1.064 \mu \mathrm{m}$ ), ${ }^{14}$ or $\chi^{(3)}=1.2 \times 10^{-13}$ esu, making it one of the possible materials for nonlinear optical device applications. Furthermore, nonresonant nonlinear optical susceptibilities of these materials may be significantly affected by the small size of nanocrystals. ${ }^{15,16}$ Here, we report on the efficient third-harmonic generation (THG) in thin films of $\mathrm{ZnO}$ leading to direct generation of UV light from an unamplified near-IR radiation of femtosecond $\mathrm{Cr}^{4+}$ :forsterite laser (1200-1300 nm).

THG is a nonlinear process in which incident, high intensity laser radiation at the frequency $\omega$, interacting with a nonlinear medium, results in the generation of an additional spectral component at the frequency $3 \omega$. For a tight, focused beam, it can be generated only on the interface between two media. ${ }^{17-20}$ Assuming that $\mathrm{TH}$ is generated in a very thin film i.e., film thickness is much less than a confocal parameter of the laser beam $b$, the power of the generated TH is given by the following formulas: ${ }^{17}$

$$
I_{3 \omega}=\frac{2304 \pi^{6}\left|\chi^{(3)}\right|}{n_{1}^{3} n_{3} \lambda_{1}^{4} c^{2}} I_{\omega}^{3} \frac{d^{2} \sin ^{2}(\Delta k d / 2)}{(\Delta k d / 2)^{2}},
$$

where $n_{1}$ and $n_{3}$ are the refractive indices of the film at the fundamental frequency and the frequency of the TH, respectively, $\lambda_{1}$ is the wavelength of the fundamental radiation, $d$ is the film thickness, $\chi^{(3)}$ is the nonlinear optical susceptibility of the film, $\Delta k$ is the wave-vector mismatch between the fundamental and TH waves in a film, and $I_{\omega}$ is the power of the incident radiation. For extremely thin films, that is, when the film thickness is much less than the coherence length, $l_{\text {coh }}=2 \pi / \Delta k$; the intensity of the TH becomes proportional to the square of the film thickness.

It is clear that, in order to achieve high conversion efficiency, one has to increase the incident intensity of the laser and use materials with the highest possible nonlinear optical coefficient $\chi^{(3)}$. An increase of the incident laser intensity above a certain threshold level will damage the material; however, wide-bandgap materials have shown resistance to the laser-induced damage up to the level of $>1 \mathrm{~J} / \mathrm{cm}^{2} \cdot{ }^{21} \mathrm{ZnO}$ is also transparent to the TH of $1200-1300 \mathrm{~nm}$ radiation, thus minimizing the probability of its damage by the intense $\mathrm{TH}$ beam. The use of femtosecond optical pulses (100 fs $\left.=10^{-13} \mathrm{~s}\right)$ results in the intensity level of $>10^{13} \mathrm{~W} / \mathrm{cm}^{2}$. Competing nonlinear optical effects, such as self-phase modulation, which typically lead to destruction of useful nonlinear optical mixing processes, are significantly reduced in thin films, because of a limited distance of light interaction with a nonlinear material.

Our sample is grown on a thin ( $1 \mathrm{~mm}$ thick) fused silica substrate by pulsed-laser deposition. The nanostructured $\mathrm{ZnO}$ films have been prepared in a mixed $\mathrm{O}_{2}-\mathrm{He}$ background gas atmosphere by conventional UV pulsed-laser (ArF laser, $\lambda=193 \mathrm{~nm}$ ) ablation. ${ }^{22}$ First, $\mathrm{ZnO}$ nanoclusters were synthesized in the gas phase, during laser-induced plume expansion and then, relatively could, stabilized, clusters were deposited on fused silica substrate. The substrate temperature was $380^{\circ} \mathrm{C}$. Resulting nanostructured films have oriented structure, with $c$ axis perpendicular to the substrate. The cluster size distribution is narrow and can be described by a Gaussian function with a maximum at $10 \mathrm{~nm}$ and a half-width of 3 $\mathrm{nm} .{ }^{23}$ The films, prepared with the optimized substrate temperature and gas partial pressures, have an intense UV excitonic luminescence and a very weak, defect-related band. The film thickness varies along the surface of a substrate, and thus allows measurements of film parameters as a function of film thickness. The maximum film thickness is slightly over $1 \mu \mathrm{m}$.

First, we confirm the homogeneity of nanoclusters along the surface of the film. We use a conventional Raman microscopy with a typical spatial resolution of about $1.5 \mu \mathrm{m}$ and spectral resolution $<3 \mathrm{~cm}^{-1}$ to measure the Raman 


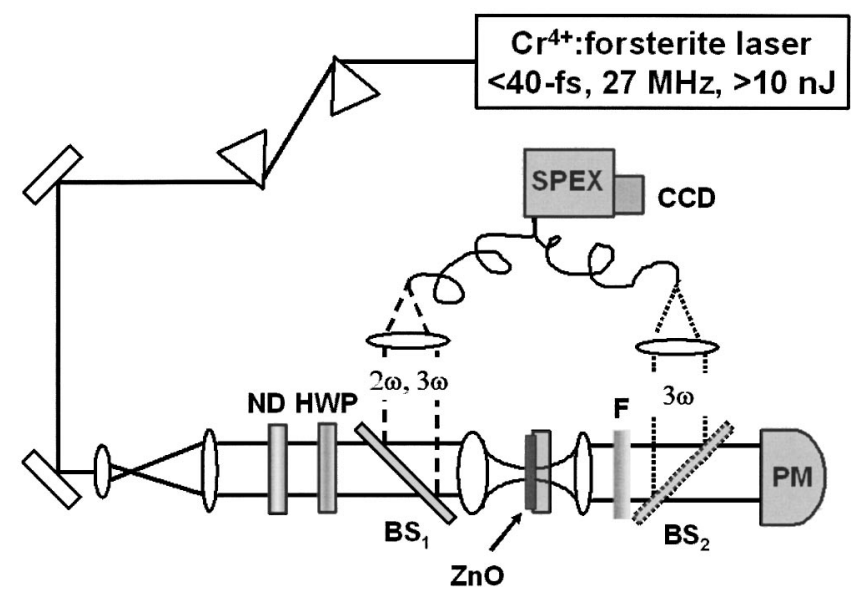

FIG. 1. Experimental setup for the THG. $\mathrm{BS}_{1,2}$ are dichroic beamsplitters, ND is a neutral density filter wheel, $F$ is a blue transmitting, near-IR absorbing filter, PM is a power meter, and HWP is a half-wave plate.

spectrum at different points on the film's surface. The major feature of this spectrum is a strong $438 \mathrm{~cm}^{-1}$ line, which has a bandwidth of about $12 \mathrm{~cm}^{-1}$, corresponding to nanoparticles of $\mathrm{ZnO}$ with a size diameter of $10 \mathrm{~nm}^{24}$ This line bandwidth remains the same over the whole surface of the film, as we would expect from our other independent measurements. $^{21}$

The experimental setup we use for our experiments is shown in Fig. 1. The laser system we use is a home-built femtosecond $\mathrm{Cr}^{4+}$ :forsterite laser. By extending the length of the laser cavity, we have recently demonstrated up to 17 $\mathrm{nJ}$ per pulse at the repetition rate of $27 \mathrm{MHz} .^{25}$ The pulse duration is typically 30-40 fs and the center wavelength is around $1250 \mathrm{~nm}$. The output of the laser is taken from the dispersive end of the cavity and is sent through the pair of prisms to compensate for the dispersion of the output coupler and the focusing lens. We use an aspheric lens (ThorLabs, Inc.) with a numerical aperture (NA) of 0.55 and a focal length of $4.5 \mathrm{~mm}$ to focus the laser radiation into the sample. The TH light is then collected from the back of the sample by using a 0.4 NA microscope objective and is redirected either into spectrometer with an attached CCD detector for spectral measurements or to a power meter. A calibrated filter is used to select the spectral radiation around $420 \mathrm{~nm}$ (the wavelength of the TH of our fundamental radiation).

By scanning the sample through the focal plane of the laser beam we can determine the confocal parameter of the beam and derive the beam waist. ${ }^{19,20}$ From these measurements, we can calculate the beam waist of the laser beam to be $(1.8 \pm 0.2) \mu \mathrm{m}$ (measured at the full width at halfmaximum). The intensity of the TH generated from the fused silica/air interface is at least four orders of magnitude weaker than one generated from the $\mathrm{ZnO} /$ air interface. We also confirm (see Fig. 2) that the intensity of the $\mathrm{TH}$ is proportional to the third power of the incoming intensity up to the maximum intensity used in our experiments $(350 \mathrm{~mW}$ of the incident power, corresponding to $13 \mathrm{~nJ}$ per pulse, which leads to the energy density of $0.5 \mathrm{~J} / \mathrm{cm}^{2}$ ). While the cascaded THG through the $\mathrm{SH}$ generation and consequent mixing of the generated radiation with the fundamental beam is possible, the efficiency of it for disoriented (in the plane of the substrate) film of $\mathrm{ZnO}$ is negligible. The latter fact is experimen-

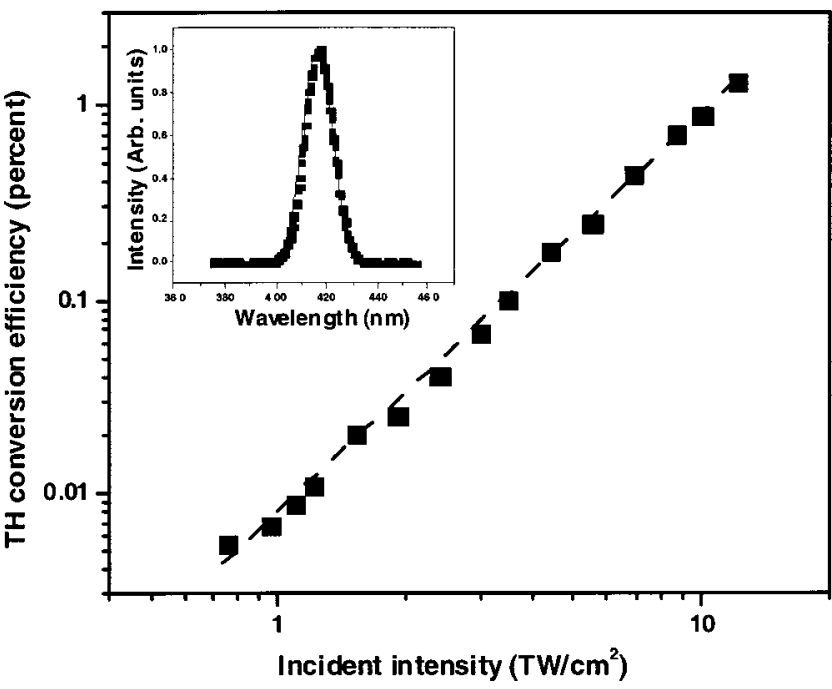

FIG. 2. Experimentally measured conversion efficiency $P_{3 \omega} / P_{\omega}$, where $P_{3 \omega}$ is the power of the generated TH and $P_{\omega}$ is the incident power of the fundamental beam. Dashed line shows a theoretically predicted quadratic dependence. Inset: experimentally measured spectrum (squares) of the $\mathrm{TH}$ at the maximum available input power. Solid line shows a theoretically predicted spectrum of the $\mathrm{TH}$ derived from the spectrum of the incident radiation.

tally confirmed by direct measurements of the $\mathrm{SH}$ power. It appears to be many orders of magnitude weaker than the power of the TH. There is no degradation of the TH intensity with time that makes us believe that the damage threshold for our $\mathrm{ZnO}$ film is higher than the maximum energy level used in our experiments. We also confirm that the spectrum of the TH (see inset of Fig. 2) remains independent on the incident power level.

We find that the efficiency of the TH generated at the maximum power level is surprisingly high. The energy of the generated TH depends on the position on the film and has strong thickness dependence. We measure up to $1.5 \mathrm{~mW}$ of the average power at the maximum incident power. When the appropriate losses on reflections and filter absorption are taken into account, we get a little more than $4.5 \mathrm{~mW}$ of generated UV light, or $1.3 \%$ conversion efficiency into the coherent TH. For a relatively thin film (approximately $200 \pm 30 \mathrm{~nm}$ thick) we can employ the Eq. (1) to calculate the value of $\chi^{(3)}$ for measured conversion efficiency. We get the value of $\chi^{(3)}=(1.4 \pm 0.7) \times 10^{-12} \mathrm{esu}$, assuming the incident intensity of $(1.2 \pm 0.2) \times 10^{13} \mathrm{~W} / \mathrm{cm}^{2}$. This value is significantly higher than earlier reported value of $\chi^{(3)}$, derived from $n_{2}$ measurements, ${ }^{14}$ which we attribute to the nanosized structure of the film. This reduced dimensionality of the film also plays a significant role in the enhancement of the second-order susceptibility $\chi^{(2)}$ in thin films of $\mathrm{ZnO} .{ }^{12} \mathrm{Re}-$ cently significant ( $\sim 500$ times) enhancement of $n_{2}$ with respect to the bulk value has been observed for polymercapped $\mathrm{ZnO}$ nanocrystals with an estimated average size of 4 nm. ${ }^{6}$ We have also tried an epitaxially grown film of $\mathrm{ZnO}$, but the energy of the TH generated from these films is more than an order of magnitude smaller than one from the nanocrystalline film of the same thickness, which confirms our hypothesis on the nanostructured nature of observed very efficient nonlinear optical conversion from our original films.

It is very important to understand the dependence of the 


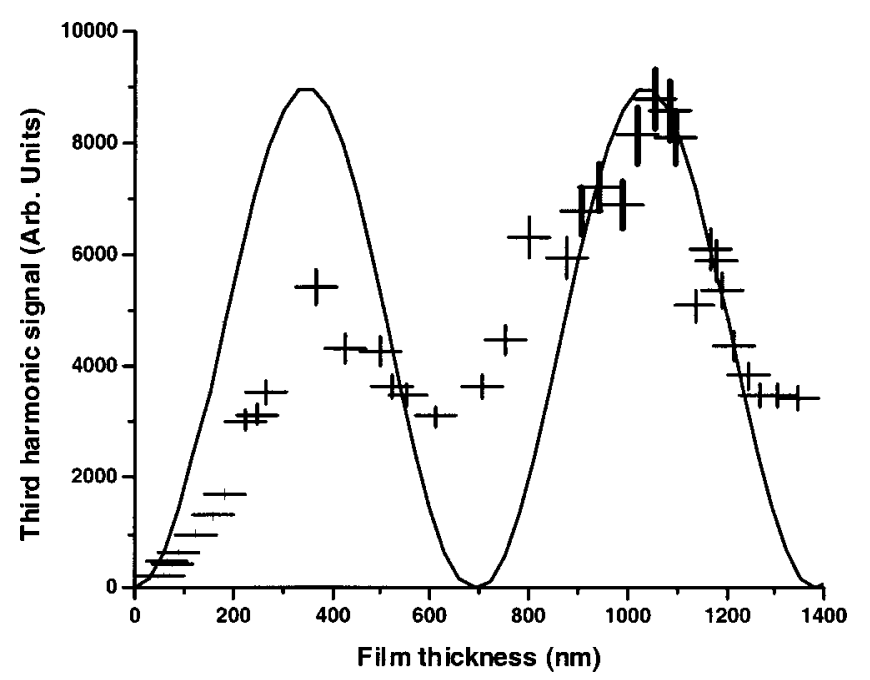

FIG. 3. Experimentally measured dependence of the TH signal on the film thickness (crosses). Experimental errors of the measured values of film thickness and power of the TH are shown as the size of crosses. Dotted line is a fit using Eq. (1).

generated TH on the thickness of this film. We scan the focal point of the laser beam across the surface of the film, simultaneously measuring the film thickness and energy of the generated TH. These data are shown as crosses in Fig. 3. The modulated dependence of the $\mathrm{TH}$ on the film thickness is clearly seen. In order to describe it, we have to include the effects of the finite coherence length for nonlinear optical interaction in the film. Since no data are available for the refractive index of $\mathrm{ZnO}$ nanocrystals, we use the data for the bulk material ${ }^{26}$ to calculate the coherence length for the TH generation process in the case of the incident light propagating along the $c$ axis of the crystal. We obtain the value of coherence length of $l_{\text {con }}^{\text {calc }}=900 \mathrm{~nm}$, which is in reasonably good agreement with the value of $l_{\mathrm{coh}}^{\mathrm{fit}}=800 \mathrm{~nm}$ obtained by fitting experimental data in Fig. 3 with a function described in Eq. (1). We note that the energy of generated TH does not drop to zero, as one would expect. However, these films are not considered to be homogeneous by all means, so that deviation of their optical properties is likely to occur. The effects of very tight focusing and light scattering in these films also may affect the phase-matching process. Nevertheless, we can conclude that under given experimental conditions, we have, indeed, obtained the maximum conversion efficiency in these films, which is of the order of percent.

We also note that no damage or any degradation to the output power of the $\mathrm{TH}$ has been observed for the highest possible conversion efficiencies. This measured maximum conversion efficiency is favorably compared to the one for cascaded THG in conventional nonlinear crystals, ${ }^{27}$ and the proposed nonlinear optical device based on a thin-film nanostructured $\mathrm{ZnO}$ film is much easier to implement since no specific angular adjustments are needed.

It is also interesting to scale our measurements down to a single nanoparticle (i.e., to the volume of about $10 \mathrm{~nm}$ ). ${ }^{3}$ Once again, assuming that the nonlinearity does not drop dramatically with the size, we obtain, under the aforementioned focusing conditions, the efficiency of the TH to be about $10^{9}$ photons/s. Partial support of these estimations has been demonstrated recently with near-field optical measurements using $800 \mathrm{~nm}$ excitation light. ${ }^{28}$

In summary, we have demonstrated that $\mathrm{ZnO}$ thin film has a very strong third-order nonlinear optical coefficient, making it possible to directly generate the third harmonic of ultrashort pulses with the efficiency of the order of percent. High efficiency of nonlinear optical conversion makes it possible to use thin films of nanostructured $\mathrm{ZnO}$ for third-order autocorrelation measurements of weak pulses ${ }^{29}$ and for characterization of single nanocrystals.

The authors would like to acknowledge support and helpful discussions with Dr. A. Sklyarov (The Advanced Analysis Facility, University of Wisconsin-Milwaukee). This work is partially supported by the NSF Grant 9984225 , and NIH Grant R21RR16282.

${ }^{1}$ M. J. Fejer, Phys. Today 47, 25 (1994).

${ }^{2}$ H. Shen, M. Wraback, J. Pamulapati, S. Liang, C. Gorla, and Y. Lu, MRS Internet J. Nitride Semicond. Res. 4S1, G3.60 (1999).

${ }^{3}$ Y. Ohta, T. Haga, and Y. Abe, Jpn. J. Appl. Phys. 36, 1040 (1997).

${ }^{4}$ D. M. Bagnall, Y. F. Chen, Z. Zhu, T. Yao, M. Y. Shen, and T. Goto, Appl. Phys. Lett. 73, 1038 (1998).

${ }^{5}$ Z. K. Tang, G. K. L. Wong, P. Yu, M. Kawasaki, A. Ohtomo, H. Koinuma, and Y. Segawa, Appl. Phys. Lett. 70, 2230 (1997).

${ }^{6}$ H. Cao, J. Y. Xu, E. W. Seelig, and R. P. H. Chang, Appl. Phys. Lett. 76, 2997 (2000).

${ }^{7}$ M. S. Wu, T. Shiosaki, and A. Kawabata, IEEE J. Quantum Electron. 25, 252 (1989).

${ }^{8}$ W. Wraback, H. Shen, S. Liang, C. R. Gorla, and Y. Lu, Appl. Phys. Lett. 74, 507 (1999).

${ }^{9}$ P. M. Lundquist, M. Jurich, J. F. Wang, H. Zhou, T. J. Marks, and G. K. Wong, Appl. Phys. Lett. 69, 901 (1996).

${ }^{10}$ W. P. Lin, P. M. Lundquist, G. K. Wong, E. D. Rippert, and J. B. Ketterson, Appl. Phys. Lett. 63, 2875 (1993).

${ }^{11}$ H. Cao, J. Y. Wu, H. C. Ong, J. Y. Dai, and R. P. H. Change, Appl. Phys. Lett. 73, 572 (1998)

${ }^{12}$ G. Wang, G. T. Kiehne, G. K. L. Wong, J. B. Ketterson, X. Liu, and R. P. H. Chang, Appl. Phys. Lett. 80, 401 (2002).

${ }^{13}$ G. P. Agrawal, Nonlinear Fiber Optics (Academic, New York, 2001).

${ }^{14}$ R. Adair, L. L. Chase, and S. A. Payne, Phys. Rev. B 39, 3337 (1989).

${ }^{15}$ G. Banfi, V. Degiorgio, and D. Ricard, Adv. Phys. 47, 447 (1998).

${ }^{16}$ L. Guo, S. Yang, C. Yang, P. Yu, J. Wang, W. Ge, and G. K. L. Wong, Appl. Phys. Lett. 76, 2901 (2000).

${ }^{17}$ J. F. Reintjes, Nonlinear Optical Parametric Processes in Liquids and Gases (Academic, Orlando, 1984).

${ }^{18}$ T. Y. F. Tsang, Phys. Rev. A 52, 4116 (1995).

${ }^{19}$ Y. Barad, H. Eisenberg, M. Horowitz, and Y. Silberberg, Appl. Phys. Lett. 70, 922 (1997).

${ }^{20}$ J. M. Schins, T. Schrama, J. Squier, G. Brakenhoff, and M. Muller, J. Opt. Soc. Am. B 19, 1627 (2002).

${ }^{21}$ W. Kautek, J. Kruger, M. Lenzner, S. Srtania, C. Spielmann, and F. Krausz, Appl. Phys. Lett. 69, 3146 (1996).

${ }^{22}$ W. Marine, L. Patrone, B. Luk'yanchuk, and M. Snetis, Appl. Surf. Sci. 154, 345 (2000).

${ }^{23}$ I. Ozerov, A. V. Bulgakov, D. Nelson, R. Castell, M. Sentis, and W. Marine, J. Phys. IV 108, 37 (2003).

${ }^{24}$ Y. Du, M. S. Zhang, J. Hong, Y. Shen, Q. Chen, and Z. Yin, Appl. Phys. A: Solid Surf. 76, 171 (2003).

${ }^{25}$ V. Shcheslavskiy, V. V. Yakovlev, and A. Ivanov, Opt. Lett. 26, 1999 (2001).

${ }^{26}$ CRC Handbook of Laser Science and Technology: Optical Materials, edited by M. J. Weber (CRC Press, Boca Raton, FL, 1997).

${ }^{27}$ X. Mu, X. Gu, M. Makarov, Y. J. Ding, L. Wang, J. Wei, and Y. Liu, Opt. Lett. 25, 117 (2000).

${ }^{28}$ J. C. Johnson, H. Q. Yan, R. D. Schaller, P. B. Peterson, P. D. Yang, and R. J. Saykally, Nano Lett. 2, 279 (2002).

${ }^{29}$ D. Meshulach, Y. Barad, and Y. Silberberg, J. Opt. Soc. Am. B 14, 2122 (1997). 